\title{
Efficient uncertainty quantification for impact analysis of human interventions in rivers
}

\author{
K.D. Berends ${ }^{\mathrm{a}, \mathrm{b}, *}$, J.J. Warmink ${ }^{\mathrm{a}}$, S.J.M.H. Hulscher ${ }^{\mathrm{a}}$ \\ a Department of Marine and Fluvial Systems, Twente Water Centre, University of Twente, P.O. Box 217, 7500 AE, Enschede, The Netherlands \\ ${ }^{\mathrm{b}}$ Department of River Dynamics and Inland Navigation, Deltares, Boussinesqweg 1, $2629 \mathrm{HV}$, Delft, The Netherlands
}

\section{A R T I C L E I N F O}

\section{Keywords:}

Impact analysis

Uncertainty

Rivers

River works

Intervention

Hydraulic modelling

Markov chain Monte Carlo

\begin{abstract}
A B S T R A C T
Human interventions to optimise river functions are often contentious, disruptive, and expensive. To analyse the expected impact of an intervention before implementation, decision makers rely on computations with complex physics-based hydraulic models. The outcome of these models is known to be sensitive to uncertain input parameters, but long model runtimes render full probabilistic assessment infeasible with standard computer resources. In this paper we propose an alternative, efficient method for uncertainty quantification for impact analysis that significantly reduces the required number of model runs by using a subsample of a full Monte Carlo ensemble to establish a probabilistic relationship between pre- and post-intervention model outcome. The efficiency of the method depends on the number of interventions, the initial Monte Carlo ensemble size and the desired level of accuracy. For the cases presented here, the computational cost was decreased by $65 \%$.
\end{abstract}

\section{Introduction}

Human interventions in rivers, usually on the scale of a hundred meters to several kilometers, are carried out all over the world to improve various, sometimes competing, river functions. Motivations for such works include flood protection (Klijn et al., 2013; Warner and van Buuren, 2011; Rijke et al., 2012) and ecological restoration (Downs and Kondolf, 2002; Buijse et al., 2002; Stewardson and Rutherfurd, 2008). Detailed physics-based models are used to quantify the impact of interventions on river systems. Lammersen et al. (2002), Bronstert et al. (2007) and Te Linde et al. (2010) studied the effect of river training works on hydraulic variables along the River Rhine. Remo et al. (2009) did a similar study for more than 100 years of river engineering along the Middle and Lower Mississipi River, and Dierauer et al. (2012) assessed the effectiveness of dike relocation (termed 'levee set back' in that paper). In 'Room for the River', a recently finished large scale flood protection program in The Netherlands which consisted of 39 projects and had a projected budget of 2.0-2.4 billion euro, impact analyses with detailed physics-based models were a key ingredient in decision support (Rijke et al., 2012). In all reported studies model accuracy was increased and tested through a calibration-validation scheme, following good modelling practice (Rykiel, 1996; van Waveren et al., 1999; Jakeman et al., 2006).

However, the inherent problem with this deterministic approach for impact analysis is that models are used to provide predictions outside measured conditions. Not only are some interventions - especially those aimed at flood protection - aimed at impact under unobserved extreme conditions, but once calibrated for a pre-intervention river system, models cannot be assumed to retain their accuracy when applied to the modified post-intervention system. Nonetheless, uncertainty is not explicitly quantified, either because there is high confidence in the physical basis of the hydraulic model (Te Linde et al., 2010) or because of limited resources (Bronstert et al., 2007).

In environmental management, there is an increasing need for policy support that is realistic about uncertainties that may impact decisions (Uusitalo et al., 2015). In model-based decision support this requires identification of potential sources of uncertainty and methods to quantify uncertainty of model output. There are many ways to categorize sources of uncertainty. One way is to distinguish between uncertainty in parameters, model input and technical implementation (Draper, 1995; Walker et al., 2003; Warmink et al., 2010; Skinner et al., 2014). In river modelling, parameter uncertainty is considered to be dominated by hydraulic roughness (Horritt and Bates, 2002; Warmink et al., 2013b), and to a lesser extent boundary conditions derived from rating curves (Pappenberger and Beven, 2006; Di Baldassarre et al., 2010) and uncertainty in geometry (van Vuren et al., 2005; Neal et al., 2015). Quantification of model output in river modelling as a function of various sources of uncertainty is carried out using variations of

\footnotetext{
* Corresponding author. Department of Marine and Fluvial Systems, Twente Water Centre, University of Twente, P.O. Box 217, 7500 AE, Enschede, The Netherlands. E-mail address: k.d.berends@utwente.nl (K.D. Berends).

URL: https://people.utwente.nl/k.d.berends (K.D. Berends).
} 


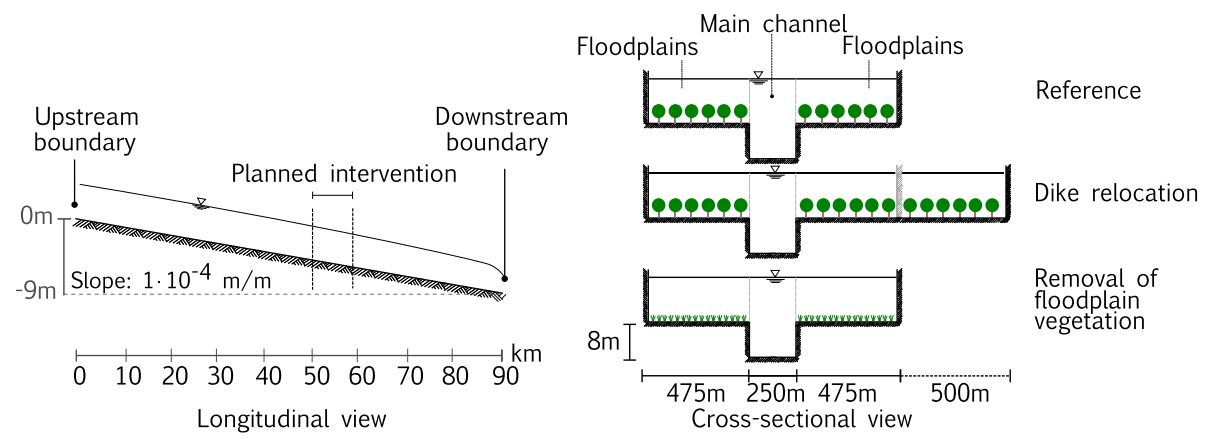

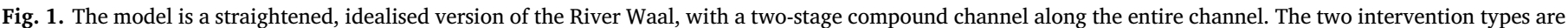
implemented between $\mathrm{km} 50$ and $\mathrm{km} 60$.

Monte Carlo simulation (Werner, 2004; Pappenberger et al., 2005, 2006; Warmink et al., 2013b; Straatsma et al., 2013; Neal et al., 2015). In catchment hydrology and climate change, probabilistic assessment of environmental impact is quantified using Monte Carlo simulation (Eckhardt et al., 2003; Breuer et al., 2006; McMichael and Hope, 2007) or multi-model approaches (Giorgi and Mearns, 2003; Tebaldi et al., 2005; Smith et al., 2009; Thirel et al., 2015). However, to our knowledge there is no literature on probabilistic impact analysis for physicsbased models in the context of a changing environmental system, either due to human intervention or natural causes. A partial explanation for this knowledge gap is that long runtimes of a detailed river models render a fully probabilistic assessment with Monte Carlo simulation arduous with standard computer resources. It becomes infeasible in the context of river intervention modelling for decision support, where designs will go through various iterations and intermediate forms before a decision to implement it can be made. Therefore, to meet the growing demand to incorporate uncertainty in decision support, a more efficient uncertainty quantification method is required.

The central question in this paper is: how can we reduce the computational investment of uncertainty quantification for impact analysis of river interventions with physics-based models? We approach this challenge by combining a hypothesis of inter-model correlation between the various models involved in impact analysis with the rigorous Bayesian approach developed in the multi-fidelity framework of Koutsourelakis (2009). The new method is tested by comparing it against a classical Monte Carlo approach.

We introduce the framework of impact assessment, the case studies and the efficient uncertainty quantification method in section 2. Results showing the output probability distributions of both the classical Monte Carlo approach as the new, efficient method are presented in section 3. Section 4 discusses potential applications of the method for decision support in river management, sensitivities and possible extensions. In section 5 we briefly summarise the method and results, and draw conclusions.

\section{Methodology}

\subsection{Definition of hydraulic river models and parameters}

A hydraulic (alternatively termed 'hydrodynamic') river model is a predictive tool made with a modelling system using data and parameters specific for a natural river system at a certain period. An example of a model is a SOBEK hydraulic model (the modelling system) for the River Waal (the natural system) as it was in the summer of 2010 (the period). Since these models are physics-based, the parameters involved generally have a clear physical interpretation. Of all the parameters in a hydraulic model, roughness coefficients are generally considered the most sensitive and uncertain ones.

Various elements in a river system generate hydraulic resistance. In the following, we consider that each roughness element has its own set of parameters, specific to the roughnes formula, and that each instance of that element inherits the same parameter values. For example, in this paper we use the Manning formula to account for friction of the roughness element 'grass', which only has the Manning coefficient as a parameter. The roughness element 'grass' is used in many places throughout the model, but with the same value of the Manning coefficient for each instance it is used. The value of the friction coefficients can be determined in various ways. For many elements, values have been estimated with empirical research (Chow, 1959). In practice, roughness parameters are often calibrated for specific cases based on often limited measurement data. However, in both cases there remains significant uncertainty in those values, the effect of which will propagate to uncertainty in model outcomes.

The spatial distribution of roughness elements in natural rivers is generally heterogeneous. In channels, roughness often comes from bed material, bed forms or structural elements like groynes. Floodplains are generally more diverse, with various vegetation species, hedges, pools and other structural features. Human intervention in a river system may change the distribution of roughness elements by removal, addition or modification of structures and roughness elements. For that reason the collection of friction parameters pre-intervention is not necessarily equal to the collection of friction parameters post-intervention.

\subsection{Case studies}

We explore two different river interventions in a low-land river. In both cases the objective is to calculate the water levels along the river at a given high and steady discharge. We refer to this discharge as the design discharge and the water levels produced during this discharge as the design water level (DWL). The use of a steady instead of unsteady conditions leads to an over-prediction of water levels by nullifying diffusive attenuation, but avoids subjectivity related to the shape of an unsteady discharge wave. The two cases are both chosen to cause a significant lowering of the DWL, but by different processes.

The first case is relocation of a dike. Many low-land rivers frequently experience water levels at or above the general level of the surrounding hinterland. Dikes are embankments specifically built to protect the hinterland against floods, but in turn constrain the river system. In the Rhine River, this has led to a so called 'technological lock-in' which necessitates continuing strengthening and raising of the dikes (Wesselink, 2007). An expensive, but effective alternative is to relocate the dikes to increase the size of the floodplains. Real-world

Table 1

Overview of roughness parameters in the model.

\begin{tabular}{llll}
\hline Roughness element & symbol & Mean & Variance \\
\hline Main channel & $n_{m}$ & 0.03 & $0.002^{2}$ \\
Brush & $n_{b}$ & 0.07 & $0.01^{2}$ \\
Grass & $n_{g}$ & 0.04 & $0.005^{2}$ \\
\hline
\end{tabular}




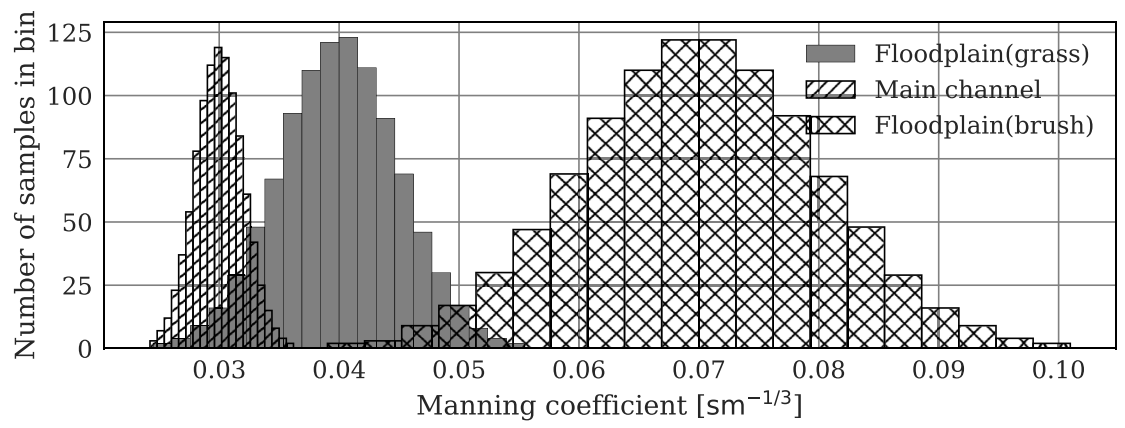

Fig. 2. Histogram of the 1000 samples for each of the three stochastic input variables.

examples of dike relocation can be found in the 'Room for the River' project in The Netherlands (Rijke et al., 2012) and along the Mississippi River (Dierauer et al., 2012). In the second case, we locally remove high-friction vegetation within the bounds of existing floodplains. Some vegetation types significantly increase water levels by offering high resistance to flow. Replacing such high-friction vegetation, such as shrubs or trees, with low-friction vegetation, like grasses, can significantly reduce flood levels.

We model both intervention cases using an idealised 1D model of the River Waal. We use a bed slope of $1 \cdot 10^{-4} \mathrm{~m} / \mathrm{m}$ and a two-stage compound cross-section consisting of a main channel and floodplains on either side. The main channel is considered to be generally smooth, while the floodplain is vegetated. To limit the number of variables, we take the floodplain to be uniformly vegetated with brushes. For the intervention of floodplain vegetation removal, we locally replace the brushes with grass. The system is schematically depicted in Fig. 1.

The model is built using SOBEK, which is a numerical modelling system that includes, amongst others, a solver for the one-dimensional shallow water (St. Venant) equations. We force the model with a steady upstream discharge of $10500 \mathrm{~m}^{3} \mathrm{~s}^{-1}$ (consistent with the discharge used to design intervention for the River Waal in the Room for the River program) and constant downstream water depth of $6 \mathrm{~m}$. We use a computational grid with a distance of $200 \mathrm{~m}$ and maximum time-step of $10 \mathrm{~min}$. The friction coefficients are resolved through Mannings formula. In this study, we assume the exact values for the coefficients unknown and normally distributed with a mean and variance based on Chow (1959). A summary of the model parameters is given in Table 1 and Fig. 2.

\subsection{Classic Monte Carlo approach to quantify uncertainty}

The main action in a hydraulic impact analysis is an inter-comparison of two distinct models which, overall, are very similar. The first model is the reference pre-intervention model that simulates the river system in its unaltered state. The second model is the post-intervention model which includes the planned intervention. The second can be seen as a modification of the first, and as such the two share all characteristics save for parts altered by the intervention. In this paper we refer to these models as $M_{x}$ with output $X$ and parameterspace $\Theta_{x}=\left\{n_{m}, n_{b}\right\}$ for the reference model and $M_{y}$ with output $Y$ and parameterspace $\Theta_{y}=\left\{n_{m}, n_{b}, n_{g}\right\}^{1}$ for the post-intervention model. The effect of the intervention can be straightforwardly calculated by subtraction: $\Delta H=Y-X$, with $X, Y$ and $\Delta H$ representing some hydraulic variable of interest. In this paper we will let $H, X$ and $Y$ be water levels, but any other output variable can be used in its place.

Since model output is not regarded as a deterministic value, but rather as a stochastic variable such that $X_{i} \sim \pi_{x}$ with $\pi_{x}$ the probability density function (pdf) of $X$, it follows that the modelled effect will be a

\footnotetext{
${ }^{1}$ The friction parameter for grass is only part of the parameter space of the second case,'vegetation removal'.
}

stochastic variable as well, i.e. $\Delta H \sim \pi_{\Delta H}$. A proven and relatively straightforward method is to approximate the output uncertainty through numerical integration by Monte Carlo simulation. The Monte Carlo method works by repeatedly sampling a possible set of parameter values from their distributions, using that set as input for a model run, and inferring probability distributions from evaluating each set in the Monte Carlo sample. In this way, we approximate $\pi_{\Delta H}$ from the ensemble $\left(\Delta H_{1}, \ldots, \Delta H_{n}\right)$ where each member is calculated from $\Delta H_{i}=Y_{i}-X_{i}$ and $n$ is sufficiently large.

Because the post-intervention model $M_{y}$ is a modification of the preintervention model, $M_{x}$, we assume their output probability functions $\pi_{x}, \pi_{y}$ are dependent. Therefore, the impact uncertainty $\pi_{\Delta H}$ depends on the covariation between $\pi_{x}, \pi_{y}$. The correlation between the two samples is preserved by using the same parameter set for generating both $Y_{i}$ and $X_{i}$. To do so, we do not sample from the respective parameterspaces $\Theta_{y}$ and $\Theta_{x}$, but rather from the union parameter space $\Theta_{x y}=\Theta_{y} \cup \Theta_{x}$. This is a vital step in constraining the uncertainty of the simulated effect, because correlation between $X$ and $Y$ will decrease the uncertainty of the effect. Additionally, it makes intuitive sense as drawing from $\Theta_{x y}$ amounts to an element-wise subtraction where the only difference between the subtracted sets is the intervention.

Generally a large number of samples is required to make sure the space of possible parameter values is adequately covered. This is especially so if the samples are drawn using a simple random draw, which is vulnerable to clusters (many draws close to each other) and gaps (unsampled regions). Alternative sampling techniques have been developed that target these problems, such as Latin Hypercube Sampling or factorial design. Here, we use a quasi-random sampling approach using the low-discrepancy sequence of Sobol', which converges much more quickly than random sampling (Saltelli et al., 2008). Using this technique, we draw 1000 samples from $\Theta_{x y}$. The results of this draw are shown in Fig. 2.

\subsection{Efficient uncertainty quantification}

The Monte Carlo method is successfully used in many fields, but its reliance on large sample size makes it prohibitively expensive in the context of intervention design, which may require iteration and evaluation of competing designs. Increasingly, faster model approximations (variously termed emulators, meta- or surrogate models) are used instead of the heavy, detailed model for resource intensive tasks like uncertainty quantification.

The applicability of such models is limited if the number of uncertain parameters is high, or if the model is used to project for unseen conditions (Razavi et al., 2012). For example, a significant system change by human intervention may easily invalidate model approximations and require retraining on the new situation. Instead of model approximates, one can also use less accurate versions of the same model by using a coarser numerical discretisation (Kennedy and O'Hagan, 2000; Koutsourelakis, 2009). This avoids many of the limitations of model approximations (Razavi et al., 2012). 


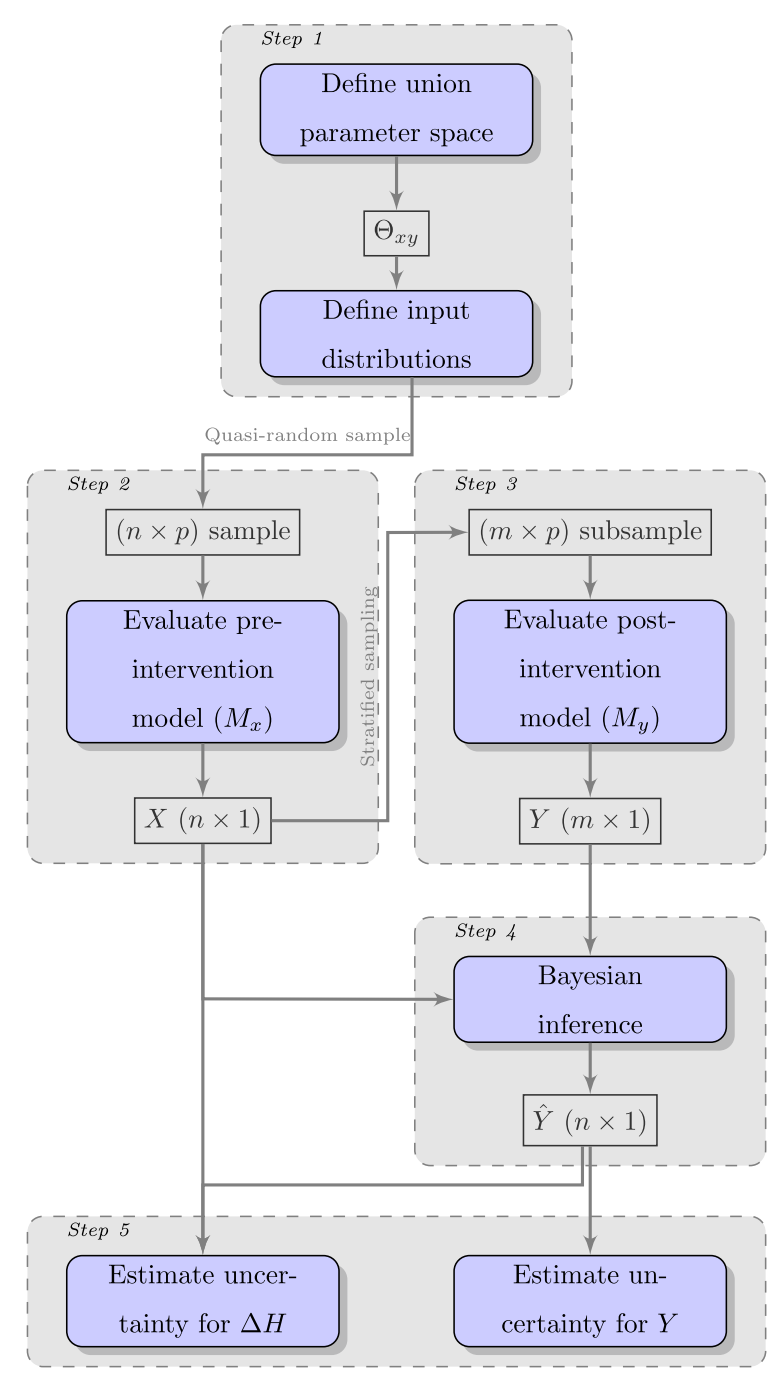

Fig. 3. Schematic overview of the efficient uncertainty estimation for post-intervention model output and impact analysis. The steps are described in detail in section 2.4. In this figure: $n$ : MC sample size; $m$ : subsample size; $p$ : number of stochastic parameters; $X$ : pre-intervention model output; $Y$ : post-intervention model output; $\hat{Y}$ : estimated post-intervention model output; $\Delta H$ : intervention effect.

Koutsourelakis (2009) showed that by exploiting the correlation between coarse and fine models, rigorous estimates of output uncertainty can be obtained. Here, we extend this concept by effectively using the pre-intervention model as an emulator for the post-intervention model. The method relies on the assumptions that (i) output of the pre- and post-intervention models $X, Y$ are not independent samples but are correlated and (ii) that we can exploit this correlation with a function $\hat{Y}=f(X)$ that allows us to approximate $Y$ and, by extension, the probability density function $\pi_{y}$. The method consists of five steps, that are repeated at each location where the uncertainty is quantified. A schematic overview of the steps is given in Fig. 3. The steps involved are the following, with $p$ uncertain parameters, Monte Carlo sample size of $n$, subsample size $m$, and $n \gg m$ :

1. Make a list of size $p$ containing all input parameters from $\Theta_{x y}$ in all models. For each parameter, specify a distribution function.

2. Generate a $(n \times p)$ sample and evaluate using $M_{x}$ to generate output $X(n \times 1)$.

3. Make a $(m \times p)$ subsample and evaluate using $M_{y}$ to generate output $Y(m \times 1)$.

4. Choose an appropriate statistical model and estimate function

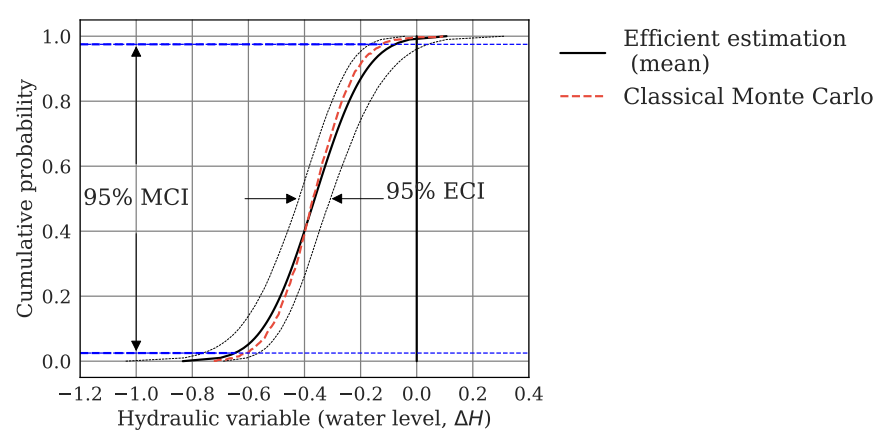

Fig. 4. The uncertainty of the model output (MCI) and the accuracy of the estimator (ECI) are both expressed as 95\% confidence intervals.

$\hat{Y}=f(X)$ given only $X$ and $Y$.

5. Use $f(X)$ to generate $\hat{Y}$ and estimate the uncertainty of $Y$ and $\Delta H$ given $X$

The first two steps follow the classical full Monte Carlo approach. We deviate from the classical approach in step three by using a subsample, instead of the full sample. The purpose of subsampling is to provide enough points along the entire range $X$ to accurately infer the function $f(X)$. We use a stratified sampling scheme by dividing the area from $[\min (X), \max (X)]$ in $m$ bins and randomly take a single sample of $X$ from each of those bins. Unless stated otherwise, we use a sample size of $n=1000$ and subsample size of $m=20$ throughout this work.

In step four we describe $Y$ in terms of $X$ and assume a general linear model if the form:

$\hat{Y}=f(X)+\varepsilon \quad$ with $\varepsilon \sim \mathscr{N}\left(0, \sigma^{2}\right)$

and

$f(X)=\alpha+\beta X$

with parameters $\alpha, \beta$ for intercept and slope and Gaussian noise term $\varepsilon$ described with a zero mean and standard deviation $\sigma$. Under the assumption of a linear relationship between $X$ and $Y$, we can infer that if $\left(\beta=1, \sigma^{2} \rightarrow 0\right)$, it follows that $\Delta H=\alpha$ with zero variance. In all other cases, the variance $V(\Delta H)>0$. From this we readily see that preserving correlation, as was discussed in section 2.3 , is a vital step to constrain uncertainty in $\Delta H$.

We infer the unknown regression parameters $\theta=\{\alpha, \beta, \sigma\}$ through Bayes' theorem. In adopting a Bayesian approach, these parameters are treated as stochastic variables. We specify prior distributions for $\theta$ reflecting our knowledge about their likely values. Then, using the $m$ observations from both $X$ and $Y$ we update these distributions through Bayes' theorem:

$p(\theta \mid Y) \propto p(\theta) p(Y \mid \theta)$

with the prior parameter distributions $p(\theta)$, the likelihood of the data given the parameters $p(Y \mid \theta)$ and the posterior likelihood of the distributions $p(\theta \mid Y)$. We obtain the posterior distribution by Markov Chain Monte Carlo (MCMC) simulation with the state-of-the-art No U-Turn Sampler (Hoffman and Gelman, 2014) within the framework of Salvatier et al. (2016), using 10000 steps, discarding half as burn-in length. We shift all the data to the origin during the inference and estimation procedure, as this was found to improve inference. For all cases we use the following priors:

- An (improper) uniform prior density on the real line for $\alpha$ and $\beta$.

- For $\sigma$ we use an inverse-gamma prior $I G(a, b)$ with $a=b=0.1$

The choice for uninformed priors reflects our lack of prior knowledge about these densities.

In step five we use the quantitative probabilistic relationship between $X$ and $Y$ from the previous step to generate full post-intervention 

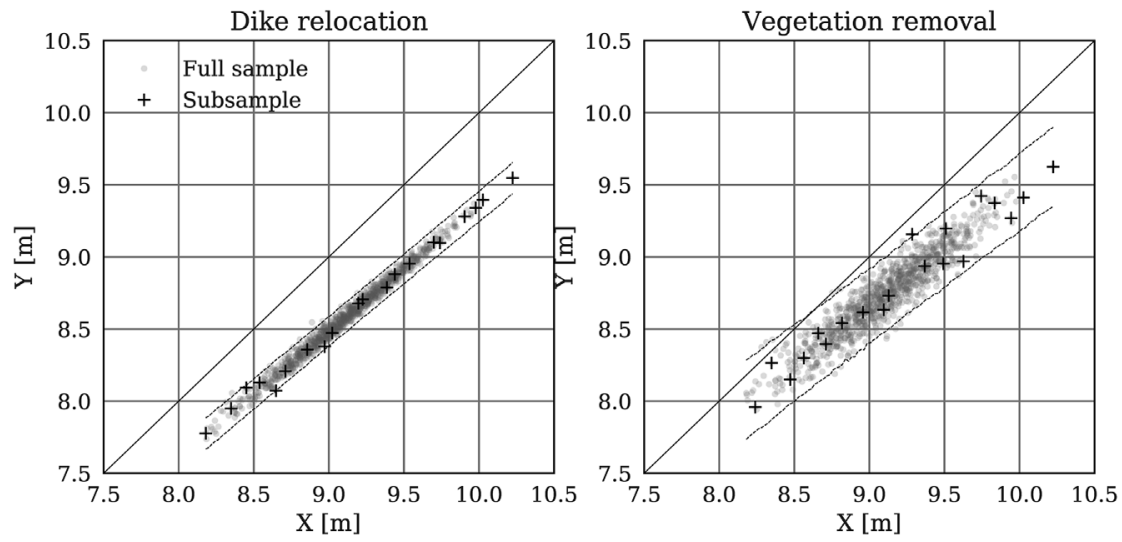

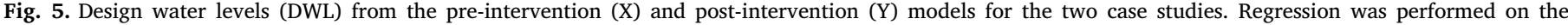
subsample $(+)$ only. The dashed lines show the confidence interval (CI) for estimated post-intervention water levels. $\hat{Y}$

samples. Since the MCMC method (after the burn-in length) samples from the joint posterior $p(\theta \mid Y)$ we can use the MCMC trace of the model parameters to generate many possible ensembles $\hat{\mathbf{Y}}$, where each $\hat{Y}$ is a simulation of the actual post-intervention water levels $Y$. We express the uncertainty of model results in terms of cumulative density functions (cdf). For the post-intervention results we show three results (see Fig. 4):

1. In dashed red, the post-intervention cdf following from the classical approach, plotted for validation of the estimation method. Of all 1000 model runs, 95\% of the ouput fell between the water levels corresponding to the 0.025 and 0.975 percentiles. We refer to this interval as the $95 \%$ MCI (model uncertainty confidence interval).

2. In solid black, the mean of the estimated post-intervention cdfs, showing the most likely location of the post-intervention cdf.

3. In dashed black lines, the $95 \%$ confidence interval of the estimated cdf. At each percentile, there is 0.95 probability that the cdf obtained with the classical approach lies between these lines. We refer to this as the $95 \%$ ECI (estimation uncertainty confidence interval). The ECI can be interpreted as an accuracy measure of the efficient estimation method.

\section{Results}

\subsection{Impact uncertainty with the classical approach}

An ensemble of 1000 parameter sets was ran with the reference model and the two intervention models. In the following we focus on the results at $50 \mathrm{~km}$ from the upper boundary, which is just upstream of the intervention where the reduction of the design water level (DWL) is at its largest. For each parameter set in the Monte Carlo sample we have two model output ensembles: the pre- and post-intervention DWLs. We have combined these results in a scatterplot (Fig. 5). Both panels show the full Monte Carlo and subsample, the regression result and the identity line to emphasize the difference between the pre- and postintervention model output. The graphs show a strong positive linear relationship between the pre-intervention $(\mathrm{X})$ and post-intervention $(\mathrm{Y})$ model outputs for both cases. This indicates that parameter sets which lead to high water level pre-intervention, also lead to high water levels post-intervention. From these graphs we can already see that the effect of the imposed dike relocation (Fig. 5, left hand panel) is likely more effective (all results well below the identity line) and less uncertain (smaller spread, higher linear correlation) than vegetation removal (Fig. 5, right hand panel). For both cases the choice for a linear statistical model (2) seems to be justified.

The distributions of pre- and post-intervention water levels and the hydraulic impact $\Delta H$ are summarised as cumulative density functions (cdfs) in Fig. 6 and numerically in Table 2. Pre-intervention, the median DWL is $9.11 \mathrm{~m}$ above the datum. ${ }^{2}$ The bounds of the $95 \%$ confidence interval are at $8.41 \mathrm{~m}$ and $9.76 \mathrm{~m}$, which leads to an MCI of $1.35 \mathrm{~m}$. After both interventions the cdfs are shifted to the left, indicating a general decrease in DWL. For dike relocation, the post-intervention median DWL is $8.58 \mathrm{~m}(-0.53 \mathrm{~m})$ with an MCI of $1.17 \mathrm{~m}(-0.18 \mathrm{~m})$. For vegetation removal, the median DWL is $8.74 \mathrm{~m}(-0.37 \mathrm{~m})$ with an MCI of $1.14 \mathrm{~m}(-0.21 \mathrm{~m})$. This means that for both interventions the post-intervention water levels are lower and less uncertain than the water levels pre-intervention. The reduced uncertainty is attributed to the linear relationship between $X$ and $Y$ having a slope $<1$; visually depicted by being non-parallel to the identity line in Fig. 5 . We posit two explanations for the cause of the negative slope. First, in the case of vegetation removal, the introduction of a new uncertain variable with relatively low variance. Second, (for both cases) a secondary effect of the Manning friction formula: lower water depths lead to higher hydraulic resistance. This secondary effect is smaller for higher water depths than lower water depths, causing a deviation from a unity slope.

Next, we look at the output uncertainty of $\Delta H$. For dike relocation, the median DWL lowering is $0.53 \mathrm{~m}$. The $95 \%$ confidence interval (MCI) is $0.23 \mathrm{~m}$, with limits at $0.4 \mathrm{~m}$ and $0.63 \mathrm{~m}$, demonstrating a slightly skewed probability distribution. For vegetation removal, the decrease in DWL is $0.37 \mathrm{~m}$ with an MCI of $0.48 \mathrm{~m}$. These results show that, within the strict bounds of the configuration used in this idealised study, vegetation removal is not only expected to be less effective in lowering DWL, but that the amount of lowering is also more uncertain. The relatively large impact uncertainty of vegetation removal can be explained from Fig. 5. Under linear correlation, the range of computed $\Delta H$ increases both with increasing variance between $X$ and $Y$, and with a deviation from the unity slope. Fig. 5 shows that vegetation removal results in larger variance, as well as a slope coefficient further from unity than is the case for dike relocation. These results show that reduction of post-intervention model output uncertainty is not necessarily a good indicator of the impact uncertainty.

\subsection{Efficient estimation of post-intervention uncertainty}

Through the Bayesian MCMC inference we obtained samples from the posterior densities of the parameters $\{\alpha, \beta, \sigma\}$, which we fed into the statistical model (1) to obtain probabilistic estimations of the model output distribution. Results are shown in Fig. 5 and summarised in Table 2. For a visual legend of the model output uncertainty (MCI) and estimation uncertainty (ECI) we refer to Fig. 4.

For the first intervention, dike relocation (Fig. 6, upper panels), we see that the mean estimated post-intervention results are virtually

\footnotetext{
${ }^{2}$ In the following, we omit the mention of the datum.
} 


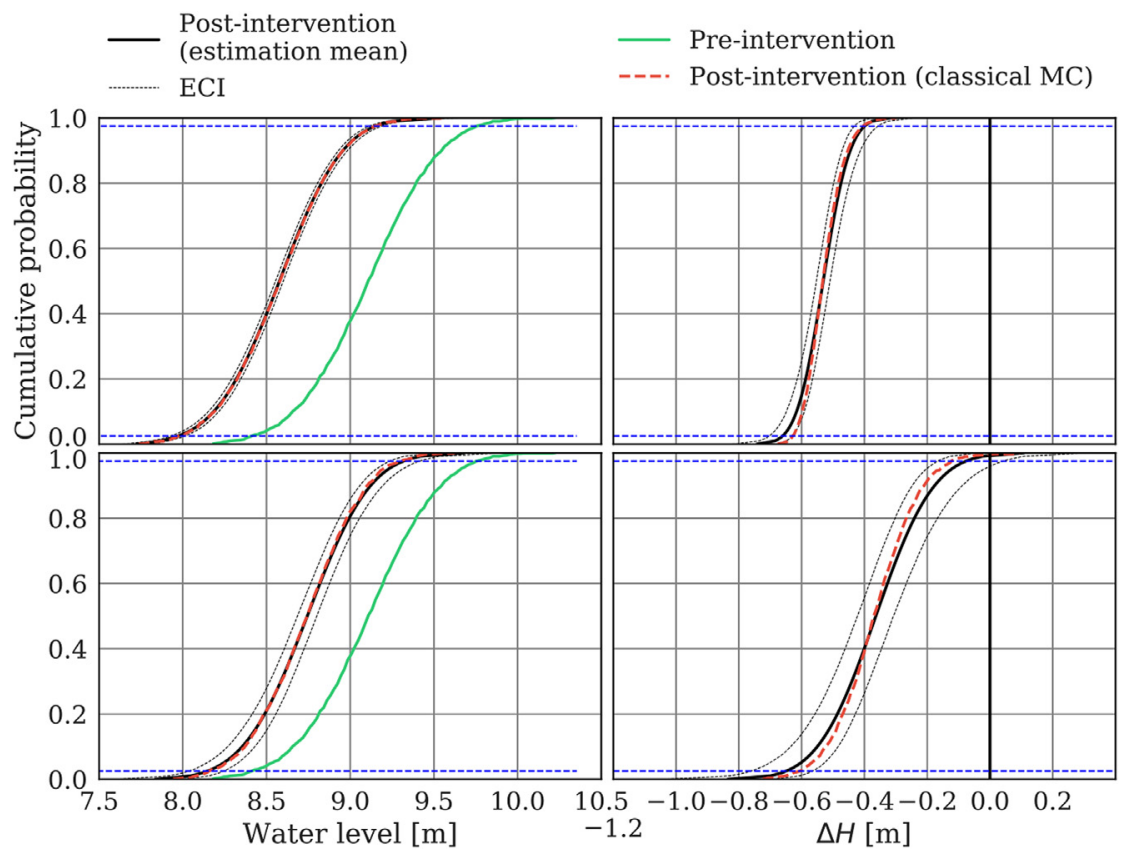

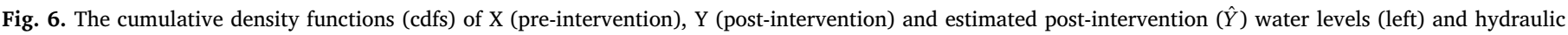
impact $\Delta H$ (right) for dike relocation (top) and vegetation removal (bottom).

Table 2

Post-intervention model uncertainty confidence interval (MCI) and estimation uncertainty confidence interval (ECI) for design water levels (DWL) and intervention effect $(\Delta H)$ from classical Monte Carlo (MC) and the efficient estimation method. All values are in meters.

\begin{tabular}{|c|c|c|c|c|c|c|}
\hline & \multicolumn{2}{|c|}{ Classical MC } & \multicolumn{4}{|c|}{ Efficient estimation } \\
\hline & \multirow[t]{2}{*}{ Median } & \multirow[t]{2}{*}{ MCI } & \multicolumn{2}{|c|}{ Median } & \multicolumn{2}{|l|}{ MCI } \\
\hline & & & Mean & ECI & Mean & ECI \\
\hline \multicolumn{7}{|c|}{ Dike Relocation } \\
\hline DWL & 8.58 & 1.17 & 8.58 & 0.05 & 1.18 & 0.11 \\
\hline$\Delta H$ & 0.53 & 0.22 & 0.53 & 0.04 & 0.26 & 0.12 \\
\hline \multicolumn{7}{|c|}{ Vegetation removal } \\
\hline DWL & 8.74 & 1.14 & 8.74 & 0.12 & 1.16 & 0.29 \\
\hline$\Delta H$ & 0.37 & 0.48 & 0.36 & 0.11 & 0.56 & 0.33 \\
\hline
\end{tabular}

identical to the classical MC results and that the ECI bounds are small, both for the water levels (upper left panel) as for the hydraulic effect (upper right panel). The median estimated effect is $0.53 \mathrm{~m}$ (classical MC: $0.53 \mathrm{~m}$ ) with an ECI of only $0.04 \mathrm{~m}$. Here, this means that there is a 95\% probability that the median effect of dike relocation obtained through classical MC lies between $0.51 \mathrm{~m}$ and $0.55 \mathrm{~m}$. Estimations were also computed for the design water levels (DWL) and the uncertainty intervals (MCI). From those results, summarised in Table 2, we see that the accuracy of the estimation method is very similar between $\Delta H$ and DWL, while the ECI increases for estimation of the MCI. A greater estimation uncertainty for the $\mathrm{MCI}$ is not surprising, as it is computed from subtracting two uncertain variables, namely the estimations $2.5 \%$ and the $97.5 \%$ percentile. Overall, we see that the estimation method is able to accurately and precisely approximate the results from the classical MC.

For the second intervention, vegetation removal (Fig. 6, lower panels), the estimated DWL and $\Delta H$ distributions agree with the results obtained through classical MC. The median DWL decrease is estimated at $0.36 \mathrm{~cm}$ with an ECI of $0.11 \mathrm{~m}$. Overall, the ECI results are larger compared to results for dike relocation. The cause for the larger ECI is the introduction of 'grass', which is a stochastic parameter not present in the pre-intervention model. The introduction of grass lead to a smaller correlation between the pre- and post-intervention model results, shown in Fig. 5. As correlation decreases, the linear model (1) is less capable to estimate the post-intervention model results. As such, the posterior distributions of the statistical model resulting from the MCMC inference are broader, which explains the larger ECI. In general, we see that if similarity between the pre- and post-intervention model decreases, the ability of the efficient method to approximate classical MC results diminishes. Nonetheless, even when the second intervention removes the entire floodplain vegetation and replaces it with a new vegetation over a length of $10 \mathrm{~km}-$ which is quite extreme - the estimation results are accurate with robust measure for estimation accuracy. The reason why there is still significant correlation between both models, even when almost $80 \%$ of the cross-sectional profile has changed, is likely due to backwater effects. This indicates that there is an important spatial dimension to uncertainty quantification, as well.

\subsection{Longitudinal intervention impact and backwater effects}

In the previous paragraphs we have shown results at $\mathrm{km} 50$ where the hydraulic effect is at its maximum. In Fig. 7 we show results obtained by repeated application of the estimation methodology for several locations along the river. The resulting trend is typical of a local river intervention. Over the length of the intervention (km $50-\mathrm{km} 60$ in both cases) equilibrium water levels are reduced. This leads to the creation of a backwater effect (of the so-called M1 type) by which the intervention impact increases from neglible at the end of the intervention to maximal at the beginning. The decrease of the DWL is then propagated upstream through another backwater effect (of the M2 type). The colors in Fig. 7 depict the probability of the interventions impact, with darker shades denoting a higher probability. For each location, we have shown results from the mean estimation cdf. Results show that the uncertainty for both interventions is greatest at the maximum effect at $\mathrm{km} 50$, with dike relocation having a greater and less uncertain effect. The effect of backwater on the uncertainty is clearly visible. From its maximum value, the uncertainty then propagates upstream through the (M2) backwater effect and decreases further from the intervention. Therefore we see that not only water levels are subject to backwater effects, but confidence intervals as well. 

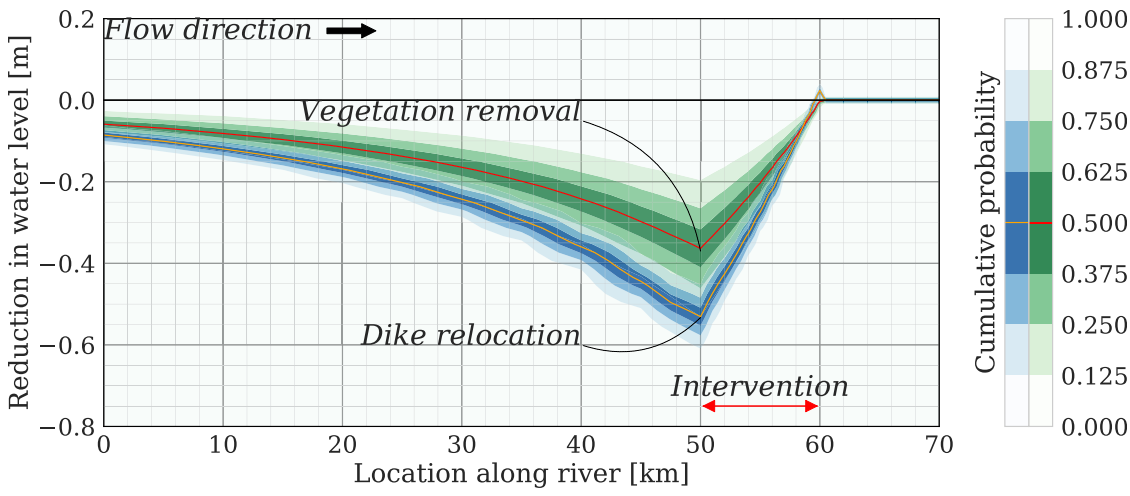

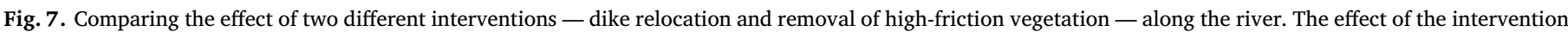
is propagated upstream through the backwater effect.

\section{Discussion}

\subsection{Application to non-idealised cases}

In this paper, we made a number of simplifications to the model and the distributions of the stochastic variables. However, in real-world cases two-dimensional models might be preferred over one-dimensional models, while the choice and distributions of stochastic variables will be based on analysis of the specific river system. Here, we discuss possible approaches to apply this method to real-world, non-idealised cases.

The uncertainty ranges in this paper were based on the tables by Chow (1959). These ranges might be less applicable for specific realworld river systems with heterogeneous floodplain vegetation and significant bed form dynamics. For example, under extreme conditions the river bed morphology may transition from river dunes to the significantly smoother upper stage plane bed (Naqshband et al., 2015; van Duin et al., 2017). In real-world cases, extensive quantification of input distributions can address these issues and lead to better informed distributions and selection of uncertainty sources (see, e.g. Straatsma and Huthoff, 2011; Warmink et al., 2013a; Neal et al., 2015).

In this article, we limited the stochastic variables to hydraulic roughness coefficients. However, additional sources may be considered including geometry (Neal et al., 2015), land-use map errors (Straatsma et al., 2013) and boundary conditions.

The act of introducing a modification to a river system itself can be uncertain as well: the intervention may not be built or develop in reallife as it was designed. This may extend to the exact location and dimensions of new embankments or uncertainty about which vegetation will naturally develop in new or modified sections of the floodplain.

Methodologically, additional uncertainty sources and input distributions can be readily incorporated, by adding the additional uncertain parameters to the union parameter space $\Theta_{x y}$. The proposed method may then be applied to any set of one-, two- or three-dimensional models. However, two remarks are made. First, in this article we considered the model output of interest (here, water level at a specific location) to be time-independent. Introduction of time dependency in the model output variable, for example by using an unsteady boundary condition, would either require iterative application of the proposed method (e.g. for each time index), or an adapted (multivariate) statistical model. If possible, the time dependency can also be eliminated by taking the average, or maximum, of the output variable. Second, the accuracy of the estimated uncertainty is limited by the correlation between the regression variables and appropriateness of the statistical model. Too great differences between pre- and post-intervention models will decrease model output correlation and increase both the uncertainty of the statistical model and the estimation uncertainty.

\subsection{The choice of a statistical model}

The efficient uncertainty quantification method described in this paper allowed us to obtain an accurate estimation of the water levels and hydraulic effect post-intervention with lower costs, for the two studied cases. However, the accuracy of this method strongly relies on the employed statistical model. In this paper we have adopted, following the principle of parsimony (Young et al., 1996), the most simple applicable model: a stochastic linear model. In both cases, the linear model was appropriate.

However, the existence of non-linear relationships between pre- and post-intervention models cannot be excluded. At low water depths, strong non-linearity in friction and vegetation models may introduce non-linearities between the regression variables. Furthermore, controlled structures, inundation of retention zones or dike breaches may introduce discontinuities. In such cases, alternative statistical models will have to be explored.

\subsection{Increasing the accuracy of the efficient uncertainty estimation}

The accuracy of the efficient uncertainty quantification method is expressed through the width of estimation uncertainty confidence interval (ECI), as shown in Fig. 6. The width of the ECI, and thus the accuracy of the estimation method is determined by two causes. First, by the width of the posterior distributions of the regression parameters $\theta=\{\alpha, \beta, \varepsilon\}$. Second, by the limited sample size $n$ of the full MC sample.

The first cause can be reduced by increasing the subsample size $m$. This will, through the MCMC inference, produce narrow posterior distributions for those parameters and a narrower ECI. To demonstrate this, we have incrementally increased the subsample size from $m=5$ to $m=500$. Results are shown in Fig. 8. We observe a sharp decrease in ECI width for small subsample sizes, followed by a slow decrease with increasing subsample size. It is not expected that the ECI will decrease to zero due to the random element $\varepsilon$ of the statistical model (1) and the large, but still finite sample size (here, $n=1000$ ) from which we generate the cdfs. ECI convergence graphs like Fig. 8 can be used to determine how many subsamples are required by starting with a low number (e.g. $m=5$ ) and incrementally increasing the subsample until the desired level of accuracy is achieved.

\subsection{Application of efficient uncertainty quantification in river management}

Quantifying uncertainty is increasingly important for decision support in environmental modelling (Uusitalo et al., 2015). Monte Carlo (MC) simulation can be used to quantify the uncertainty of model output when model input and parameters are uncertain. However, as a river system changes due to natural of human causes, the uncertainty quantification needs to be updated for the new system. This adds to an already heavy and often infeasible computational burden. 


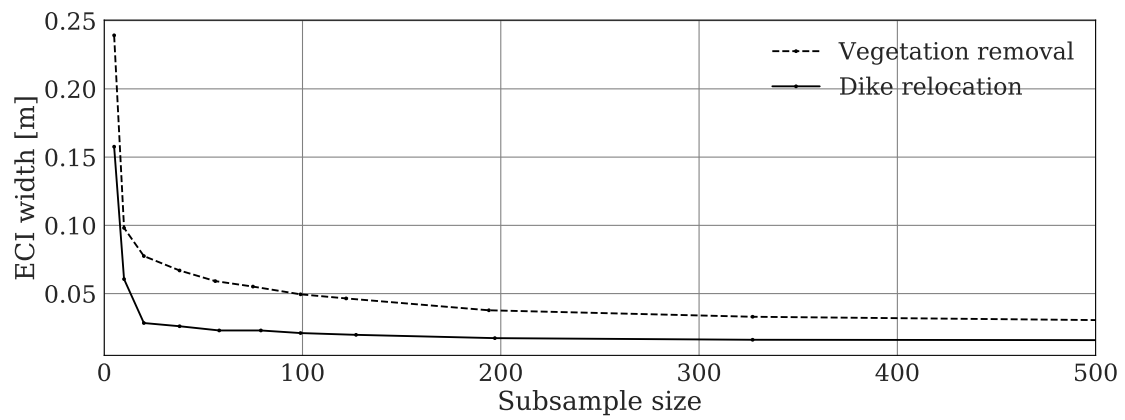

Fig. 8. The width of the estimation uncertainty confidence interval (ECI) of the median post-intervention water levels as function of subsample size.

In this paper we have shown for two idealised cases that similarity between two models can be exploited to significantly reduce the cost of MC simulations, if a full MC for a reference model is available. In intervention design, this method can be used to rapidly and robustly quantify the probabilistic intervention effects while prototyping various designs.

The effective method offers three main benefits. First, it reduces the computational cost. In terms of effectiveness, the proposed method reduced the number of model runs by $E=1-\frac{n+k m}{n+k n}$, assuming the runtime is equal for all models involved, with $k$ the number of interventions or otherwise changed systems. For the case presented in this paper ( $n=1000, m=20, k=2$ ) the effectiveness is approximately $65 \%$, not accounting for the statistical analysis. In our study this additional effort was in the order of minutes, although no additional effort was spend on optimisation of this process.

Second, the models runs performed during the efficient uncertainty quantification can be re-used to increase accuracy if more resources become available for a larger subsample or even a full sample. Following above formula, the efficiency of the method will then decrease accordingly to zero for a full sample. Finally, the method is non invasive (it requires no changes to model code) and model softwareindependent.

\section{Conclusion}

Detailed physics-based models are increasingly used to support policy in river management. These models are known to be sensitive to uncertain input, but quantifying the uncertainty of model outcome is very computer intensive, necessitating methods to decrease the computational burden.

In this paper we have proposed an alternative, efficient uncertainty estimation method that operates on the key insight that both models involved in impact analysis (a pre- and a post-intervention model) are extremely similar in most ways - apart from the obvious system changes introduced by the intervention. This similarity is manifested in a correlation of model outcomes, which can be first approximated and then exploited, using only a subset of the full post-intervention ensemble. We describe the correlation between the pre- and post-intervention models probabilistically using Bayesian regression within a Markov-Chain Monte Carlo framework. In using a Bayesian approach, we are not only able to accurately estimate the impact uncertainty, but obtain a robust measure for the estimation accuracy as well.

We compared probability density functions of post-intervention water levels and of the intervention impact computed with the efficient estimation method against a classical Monte Carlo approach for two disparate idealised river interventions aimed at decreasing flood levels during high discharge: one (dike relocation) aimed at greatly increasing the cross-sectional area, the other (vegetation removal) at fundamentally changing floodplain configuration. In both cases, uncertainty in model output is introduced by uncertainty in the friction parameters.

Inspection of the results show interesting similarities and differences between the two intervention cases. For both cases, we observed a reduction in model output uncertainty after intervention. However, the impact uncertainty of the intervention is different. In particular, fundamentally changing the floodplain configuration by introducing a new uncertain variable (here: a new vegetation type) leads to a lower correlation between pre- and post-intervention model outcome and consequently to a greater uncertainty in intervention impact.

Although further research is required to generalize our results for specific interventions and more complex cases, the results underline the importance of uncertainty quantification for models that support decision making.

The efficient uncertainty quantification method introduced in this paper was used to obtain accurate estimations of post-intervention water levels and impact. Results show good agreement between the classical approach and the new, efficient method even at a subsample size of 20 model runs. The accuracy of the estimated uncertainty depends on the correlation between the pre- and post-intervention model output, as well as the appropriateness of the statistical model. In our cases, the computational costs was reduced by $65 \%$, compared to full Monte Carlo simulation.

\section{Acknowledgments}

This research is part of the RiverCare research programme, supported by the Dutch Technology Foundation TTW (project-number 13520), which is part of the Netherlands Organisation for Scientific Research (NWO), and which is partly funded by the Ministry of Economic Affairs under grant number P12-14 (Perspective Programme). This research has benefited from cooperation within the Netherlands Centre for River studies (www.ncr-web.org). We thank three anonymous reviewers for their constructive comments and suggestions for improvement.

\section{Appendix A. Software availability}

The method described in this paper was implemented in Python 2.7. The implementation is available for download from hdl:10411/ GCS6HE, including test data for the cases described in this paper. We make use of the Anaconda distribution, freely available from https:// www.continuum.io/, and the packages SAlib (Herman and Usher, 2017) for quasi-random sampling and pyMC3 (Salvatier et al., 2016) for NUTS MCMC. The numerical model used to generate data is SOBEK, version 3.4.2. It is part of the Delft3D Flexible Mesh software suite. For information on availability and licensing we refer to https://www. deltares.nl/en/software/sobek/. Full reproduction data, including the hydraulic models themselves, is available from the corresponding author of this paper upon request.

\section{References}

Di Baldassarre, G., Schumann, G., Bates, P.D., Freer, J.E., Beven, K.J., 2010. Flood-plain mapping: a critical discussion of deterministic and probabilistic approaches. Hydrol. Sci. J. 55, 364-376. http://dx.doi.org/10.1080/02626661003683389. 
Breuer, L., Huisman, J.A., Frede, H.G., 2006. Monte Carlo assessment of uncertainty in the simulated hydrological response to land use change. Environ. Model. Assess. 11, 209-218. http://dx.doi.org/10.1007/s10666-006-9051-9.

Bronstert, A., Bárdossy, A., Bismuth, C., Buiteveld, H., Disse, M., Engel, H., Fritsch, U., Hundecha, Y., Lammersen, R., Niehoff, D., Ritter, N., 2007. Multi-scale modelling of land-use change and river training effects on floods in the rhine basin. River Res. Appl. 23, 1102-1125. URL: https://doi.org/10.1002/rra.1036.

Buijse, A.D., Coops, H., Staras, M., Jans, L.H., van Geest, G.J., Grift, R.E., Ibelings, B.W., Oosterberg, W., Roozen, F.C.J.M., 2002. Restoration strategies for river floodplain along large lowland rivers in europe. Freshw. Biol. 47, 889-907. http://dx.doi.org/ 10.1046/j.1365-2427.2002.00915.x.

Chow, V.T., 1959. Open-channel Hydraulics. McGraw-Hill, New York.

Dierauer, J., Pinter, N., Remo, J.W., 2012. Evaluation of levee setbacks for flood-loss reduction, middle Mississippi river, USA. J. Hydrol. 450-451, 1-8. http://dx.doi.org/ 10.1016/j.jhydrol.2012.05.044.

Downs, P.W., Kondolf, G.M., 2002. Post-project appraisals in adaptive management of river channel restoration. Environ. Manag. 29, 477-496.

Draper, D., 1995. Assessment and propagation of model uncertainty. J. Roy. Stat. Soc. B (Methodological) 57, 45-97. URL: http://links.jstor.org/sici?sici=0035 9246\%2801995\%2957\%3A1\%3C45\%3AAAPOMU\%3E2.0.C0\%3B2-7.

van Duin, O.J.M., Hulscher, S.J.M.H., Ribberink, J.S., Dohmen-Janssen, C.M., 2017. Modeling of spatial lag in bed-load transport processes and its effect on dune morphology. J. Hydraul. Eng. 143, 04016084. http://dx.doi.org/10.1061/(asce)hy.1943 7900.0001254

Eckhardt, K., Breuer, L., Frede, H.G., 2003. Parameter uncertainty and the significance of simulated land use change effects. J. Hydrol. 273, 164-176.

Giorgi, F., Mearns, L.O., 2003. Probability of regional climate change based on the reliability ensemble averaging (REA) method. Geophys. Res. Lett. 30. http://dx.doi. org/10.1029/2003GL017130.

Herman, J., Usher, W., 2017. Salib: an open-source python library for sensitivity analysis. J. Open Source Softw. 2. http://dx.doi.org/10.21105/joss.00097.

Hoffman, M.D., Gelman, A., 2014. The no-u-turn sampler: adaptively setting path lengths in Hamiltonian Monte Carlo. J. Mach. Learn. Res. 15, 1593-1623.

Horritt, M.S., Bates, P.D., 2002. Evaluation of 1d and 2d numerical models for predicting flood inundation. J. Hydrol. 268, 87-99. http://dx.doi.org/10.1016/S0022-1694(02) 00121-X.

Jakeman, A.J., Letcher, R.A., Norton, J.P., 2006. Ten iterative steps in development and evaluation of environmental models. Environ. Model. Software 21, 602-614. http:// dx.doi.org/10.1016/j.envsoft.2006.01.004.

Kennedy, M.C., O'Hagan, A., 2000. Predicting the output from a complex computer code when fast approximations are available. Biometrika 87, 1-13. URL: https://doi.org/ 10.1093/biomet/87.1.1.

Klijn, F., de Bruin, D., de Hoog, M.C., Jansen, S., Sijmons, D.F., 2013. Design quality of room-for-the-river measures in The Netherlands: role and assessment of the quality team (q-team). Int. J. River Basin Manag. 11, 287-299. http://dx.doi.org/10.1080/ 15715124.2013.811418.

Koutsourelakis, P.S., 2009. Accurate uncertainty quantification using inaccurate computational models. SIAM J. Sci. Comput. 31, 3274-3300. URL: https://doi.org/10. $1137 / 080733565$.

Lammersen, R., Engel, H., van de Langemheen, W., Buiteveld, H., 2002. Impact of river training and retention measures on flood peaks along the rhine. J. Hydrol. 267, 115-124. URL: https://doi.org/10.1016/S0022-1694(02)00144-0.

McMichael, C.E., Hope, A.S., 2007. Predicting streamflow response to fire-induced landcover change: implications of parameter uncertainty in the MIKE SHE model. J. Environ. Manag. 84, 245-256. http://dx.doi.org/10.1016/j.jenvman.2006.06.003.

Naqshband, S., van Duin, O., Ribberink, J., Hulscher, S., 2015. Modeling river dune development and dune transition to upper stage plane bed. Earth Surf. Process. Landforms 41, 323-335. http://dx.doi.org/10.1002/esp.3789.

Neal, J.C., Odoni, N.A., Trigg, M.A., Freer, J.E., Garcia-Pintado, J., Mason, D.C., Wood, M., Bates, P.D., 2015. Efficient incorporation of channel cross-section geometry un certainty into regional and global scale flood inundation models. J. Hydrol. 529, 169-183. http://dx.doi.org/10.1016/j.jhydrol.2015.07.026.

Pappenberger, F., Beven, K.J., 2006. Ignorance is bliss: or seven reasons not to use uncertainty analysis. Water Resour. Res. 42. http://dx.doi.org/10.1029/ 2005WR004820.

Pappenberger, F., Beven, K., Horritt, M., Blazkova, S., 2005. Uncertainty in the calibration of effective roughness parameters in hec-ras using inundation and downstream level observations. J. Hydrol. 302, 46-69. http://dx.doi.org/10.1016/j.jhydrol.2004.06. 036.

Pappenberger, F., Matgen, P., Beven, K.J., Henry, J.B., Pfister, L., de Fraipont, P., 2006. Influence of uncertain boundary conditions and model structure on flood inundation predictions. Adv. Water Resour. 29, 1430-1449. http://dx.doi.org/10.1016/j. advwatres.2005.11.012.

Razavi, S., Tolson, B.A., Burn, D.H., 2012. Review of surrogate modeling in water resources. Water Resour. Res. 48, W07401. URL: https://doi.org/10.1029/ 2011 WR011527.
Remo, J.W., Pinter, N., Heine, R., 2009. The use of retro- and scenario-modeling to assess effects of $100+$ years river of engineering and land-cover change on middle and lower Mississippi river flood stages. J. Hydrol. 376, 403-416. http://dx.doi.org/10. 1016/j.jhydrol.2009.07.049.

Rijke, J., van Herk, S., Zevenbergen, C., Ashley, R., 2012. Room for the river: delivering integrated river basin management in The Netherlands. Int. J. River Basin Manag. 10, 369-382. http://dx.doi.org/10.1080/15715124.2012.739173.

Rykiel, E.J., 1996. Testing ecological models: the meaning of validation. Ecol. Model. 90, 229-244. http://dx.doi.org/10.1016/0304-3800(95)00152-2.

Saltelli, A., Chan, K., Scott, E.M., 2008. Sensitivity Analysis. John Wiley and Sons Ltd.

Salvatier, J., Wiecki, T.V., Fonnesbeck, C., 2016. Probabilistic programming in python using PyMC3. PeerJ Computer Sci. 2, e55. http://dx.doi.org/10.7717/peerj-cs.55.

Skinner, D.J.C., Rocks, S.A., Pollard, S.J.T., 2014. A review of uncertainty in environmental risk: characterising potential nature, locations and levels. J. Risk Res. 14, 195-219. http://dx.doi.org/10.1080/13669877.2013.794150.

Smith, R.L., Tebaldi, C., Nychka, D., Mearns, L.O., 2009. Bayesian modeling of uncertainty in ensembles of climate models. J. Am. Stat. Assoc. 104, 97-116. http://dx. doi.org/10.1198/jasa.2009.0007.

Stewardson, M., Rutherfurd, I., 2008. Conceptual and mathematical modelling in river restoration. In: River Restoration: Managing the Uncertainty in Restoring Physical Habitat. John Wiley and Sons Ltd., pp. 61-78. https://doi.org/10.1002/ 9780470867082.ch5.

Straatsma, M., Huthoff, F., 2011. Uncertainty in 2d hydrodynamic models from erros in roughness parameterization based on aerial images. Phys. Chem. Earth 36, 324-334. http://dx.doi.org/10.1016/j.pce.2011.02.009.

Straatsma, M.W., van der Perk, M., Schipper, A.M., de Nooij, R.J.W., Leuven, R.S.E.W., Huthoff, F., Middelkoop, H., 2013. Uncertainty in hydromorphological and ecological modelling of lowland river floodplains resulting from land cover classification errors. Environ. Model. Software 42, 17-29. URL: https://doi.org/10.1016/j.envsoft.2012. 11.014.

Te Linde, A., Aerts, J., Kwadijk, J., 2010. Effectiveness of flood management measures on peak discharges in the rhine basin under climate change. J Flood Risk Manag. 3, 248-269. http://dx.doi.org/10.1111/j.1753-318X.2010.01076.x.

Tebaldi, C., Smith, R.L., Nychka, D., Mearns, L.O., 2005. Quantifying uncertainty in projections of regional climate change: a bayesian approach to the analysis of multimodel ensembles. J. Clim. 18, 1524-1540. http://dx.doi.org/10.1175/JCLI3363.1.

Thirel, G., Andréassian, V., Perrin, C., Audouy, J.N., Berthet, L., Edwards, P., Folton, N., Furusho, C., Kuentz, A., Lerat, J., Lindström, G., Martin, E., Mathevet, T., Merz, R., Parajka, J., Ruelland, D., Vaze, J., 2015. Hydrology under change: an evaluation protocol to investigate how hydrological models deal with changing catchments. Hydrol. Sci. J. 60, 1184-1199. http://dx.doi.org/10.1080/02626667.2014.967248.

Uusitalo, L., Lehikoinen, A., Helle, I., Myrberg, K., 2015. An overview of methods to evaluate uncertainty of deterministic models in decision support. Environ. Model. Software 63, 24-31. http://dx.doi.org/10.1016/j.envsoft.2014.09.017.

van Vuren, S., de Vriend, H.J., Ouwerkerk, S., Kok, M., 2005. Stochastic modelling of the impact of flood protection measures along the river Waal in The Netherlands. Nat. Hazards 36, 81-102 doi:s11069-004-4543-x.

Walker, W.E., Harremoës, P., Rotmans, J., van der Sluijs, J.P., van Asselt, M.B.A., Janssen, P., von Krauss, M.P.K., 2003. Defining uncertainty: a conceptual basis for uncertainty management in model-based decision support. Integrated Assess. 4, 5-17. http://dx doi.org/10.1076/iaij.4.1.5.16466.

Warmink, J., Janssen, J., Booij, M., Krol, M., 2010. Identification and classification of uncertainties in the application of environmental models. Environ. Model. Software 25, 1518-1527. http://dx.doi.org/10.1016/j.envsoft.2010.04.011.

Warmink, J., Booij, M., van der Klis, H., Hulscher, S., 2013a. Quantification of uncertainty in design water levels due to uncertain bed form roughness in the Dutch rivier waal. Hydrol. Process. 27, 1646-1663. http://dx.doi.org/10.1002/hyp.9319.

Warmink, J.J., Straatsma, M.W., Huhoff, F., Booij, M.J., Hulscher, S.J.M.H., 2013b. Uncertainty of design water levels due to combined bed form and vegetation roughness in the Dutch river waal. J Flood Risk Manag. 6, 302-318. http://dx.doi. org/10.1111/jfr3.12014.

Warner, J., van Buuren, A., 2011. Implementing room for the river: narratives of success and failure in kampen, The Netherlands. Int. Rev. Adm. Sci. 77, 779-801. http://dx. doi.org/10.1177/0020852311419387.

van Waveren, R.H., Groot, S., Scholten, H., van Geer, F.C., Wösten, J.H.M., Koeze, R.D., Noort, J.J., 1999. Good Modelling Practice Handbook. STOWA report. 99-05, RIZAreport 99.036

Werner, M.G.F., 2004. A comparison of flood extent modelling approaches through constraining uncertainties on gauge data. Hydrol. Earth Syst. Sci. 8, 1141-1152.

Wesselink, A.J., 2007. Flood safety in The Netherlands: the Dutch response to hurricane katrina. Technol. Soc. 29, 239-247. http://dx.doi.org/10.1016/j.techsoc.2007.01. 010.

Young, P., Parkinson, S., Lees, M., 1996. Simplicity out of complexity in environmental modelling: Occam's razor revisited. J. Appl. Stat. 23, 165-210. http://dx.doi.org/10. $1080 / 02664769624206$. 